\title{
UMA PLATAFORMA DE SIMULAÇÃO VISUAL EM 3D ORIENTADA PARA O CICLO DE VIDA DAS ENTIDADES
}

\section{A 3D VISUAL SIMULATION PLATFORM ORIENTED TO THE ENTITIES'S LIFE CYCLE}

\author{
Mário Jorge Ferreira de Oliveira \\ Professor Associado II, PhD. \\ e-mail: mario_jo@pep.ufrj.br
}

\author{
Francisco Santos Sabbadini \\ Doutorando em Engenharia de Produção \\ e-mail: sabba@pep.ufrj.br \\ Ilan Chamowitz \\ Doutorando em Engenharia de Produção \\ e-mail: ilan@pep.ufrj.br
}

\author{
Universidade Federal do Rio de Janeiro - COPPE \\ Cidade Universitária - C.T. \\ Bloco F, Sala 105 \\ 21945-970 - Rio de Janeiro - RJ \\ Telefone: (21) 2562-8244
}




\title{
RESUMO
}

O objetivo do artigo é apresentar uma plataforma para simulação visual em 3D, desenvolvida no Programa de Engenharia de Produção da COPPE na Universidade Federal do Rio de Janeiro. O sistema foi projetado, originalmente, como uma ferramenta para auxiliar alunos do curso de simulação a conduzir um experimento completo com um modelo de simulação a eventos discretos. Este artigo faz uma revisão da literatura e, a partir de uma perspectiva histórica, aponta as origens e a direção de desenvolvimento de novas pesquisas neste campo. Uma avaliação do cenário atual é realizada assim como são descritas as oportunidades de desenvolvimentos futuros nesta área emergente no campo da simulação a eventos discretos.

Palavras-Chave: Pesquisa Operacional, Modelagem, Simulação a eventos discretos, Diagrama de Ciclo de entidades, Simulação Visual em 3D.

\begin{abstract}
The objective of this paper is to present a 3D visual simulation platform, developed at the production engineering program of COPPE in the Federal University of Rio de Janeiro. The system was projected, originally, as a tool to let Msc students carry out a full experiment with a discrete-event simulation model. This paper makes a literature review from a historical perspective and points to the origins and the directions development of this new research field. An evaluation of the state of art is carried through as well as the description of the chances of future developments in this emergent area.
\end{abstract}

Key-words: Operational Research, Modeling, Discrete event simulation, Activity Cycle Diagram, 3D visual simulation.

\section{INTRODUÇÃO}

O desenvolvimento de recursos de computação nas últimas décadas resultou em um avanço significativo, nas mais diversas áreas do conhecimento. A produção de computadores, com maior poder de processamento e custo reduzido, torna viável a utilização de modelos sofisticados para análise e solução de problemas complexos. O progresso tecnológico no campo da computação torna possível a obtenção de recursos gráficos e ambientes virtuais e novas possibilidades de aplicações práticas, em várias áreas, surgem a cada ano (LAGERGREN, 2002).

A Pesquisa Operacional (PO) é um campo onde se tem produzido uma grande quantidade de novas ferramentas para melhorar a maneira de estruturar e pensar sobre 
problemas existentes em várias áreas do conhecimento. Os métodos de PO são usualmente desenvolvidos por meio de modelos formais e abstratos. Modelagem é a arte de construir e utilizar modelos como ferramenta de análise. Uma revisão abrangente sobre o surgimento dos primeiros modelos de simulação na área de saúde é apresentada por De Oliveira (2000).

O hospital, como uma entidade social voltada para a atenção e cuidado aos pacientes é conhecido, há muito tempo, como uma área de aplicação para os métodos de PO. Modelos para avaliar a utilização de recursos na operação de sistemas de saúde são apresentados na literatura a partir da década de 60 (TUNICLIFE-WILSON, 1980). Esse tipo de abordagem tem sido direcionado para previsão de demanda, marcação de consultas, gerenciamento de filas e escala de pessoal (BENNETT, 1994). Aplicações recentes podem ser encontradas na gestão da capacidade de atendimento no Instituto Nacional do Câncer (GONÇALVES, 2004), em sistema de admissão de emergência hospitalar (SABBADINI, 2005), e no dimensionamento do serviço de atendimento móvel de urgência (GARCIA, 2006).

Atualmente, podemos encontrar aplicações sofisticadas, apoiadas em recursos tecnológicos avançados. A visualização da simulação vem sendo estudada por mais de uma década. O desenvolvimento de plataformas visuais interativas conduziu ao estudo de métodos voltados para a solução de problemas complexos. Um projeto originalmente desenvolvido por Balci et al (1997) foi pioneiro no sentido de propor, a um custo bastante elevado, protótipos de uma plataforma de simulação visual em 2D. Uma plataforma para a simulação visual em 3D (DE OLIVEIRA, 1999) e avanços recentes são apresentados e discutidos neste artigo.

\section{ORIGENS E EVOLUÇÃO}

O avanço da modelagem visual na representação de objetos e a produção de equipamentos que suportem as exigências na representação visual em 3D representaram um salto significativo. Dois conceitos citados na literatura devem ser destacados dada a sua relevância na área de simulação visual: "Visual Interactive Simulation” (VIS), descrito por Hurrion (1976) e Visual Interactive Modeling (VIM) por Bell e O`Keefe (1987). Segundo Wagner et al. $(1996 ; 1997)$ os dois conceitos estão voltados para a produção de ferramentas que visam possibilitar a condução dos experimentos de simulação de uma forma mais amigável. 
A principal característica de VIS é a sugestão de uma visualização de dados intermediários e a capacidade de interação com o modelo durante a realização da simulação de modo integrado, oferecendo ao usuário mecanismos para que este possa compreender e melhorar o sistema em estudo. Com relação a VIM, a idéia original era aumentar a produtividade do modelador, com ênfase na construção do modelo através dos recursos gráficos disponíveis naquela época. Ao detectarem esta diferença entre construção interativa de um modelo e a experimentação interativa do modelo construído, os autores sugerem a possibilidade de um ambiente VISM, incorporando tanto recursos de VIS quanto de VIM.

O termo "Visual Simulation Environment" (VSE) surgiu no período compreendido entre 1983 e 1995, a partir de um projeto da marinha americana, onde se desenvolveu um método de simulação a eventos discreto de propósito geral, para a análise de problemas complexos. Segundo Balci et al (1997), o sistema é composto de quatro módulos: Um editor, um simulador, uma ferramenta de análise dos resultados e um tutorial.

O projeto VSE resultou de um processo evolutivo desenvolvido a partir de dois outros projetos: "Simulation Model Development Environment" (SMDE) e "Visual Simulation Support Environment" (VSSE). Devido ao incremento da complexidade dos modelos de simulação e à necessidade de reduzir o ciclo de vida no desenvolvimento destes, iniciou-se, a partir de 1983, o projeto SMDE, voltado para a pesquisa de problemas complexos, no sentido de prover a integração de ferramentas baseadas em computador para aumentar a produtividade, a qualidade e reduzir o tempo de construção dos modelos (BALCI, 1997).

\section{DIAGRAMA DE CICLO DE ATIVIDADES (DCA)}

O conceito de ciclo de atividades é apresentado por Paul (1993) como um método de formulação de um problema em termos de fluxo lógico das atividades num sistema. Nesse sentido um diagrama pode ser utilizado como base para a codificação computacional de um modelo em qualquer linguagem utilizando qualquer paradigma como, por exemplo, eventos, atividades ou processos.

Um Diagrama de Ciclo de Atividades (DCA) é uma descrição do comportamento dinâmico das atividades de um sistema. As entidades envolvidas num modelo de simulação 
interagem durante o tempo de execução do fluxo lógico do modelo de simulação discreta. Essas interações podem ser descritas através de um DCA.

Segundo Paul (1993) um ciclo de vida é definido para cada tipo de atividade, havendo uma alternância entre filas e atividades para o DCA de qualquer uma das entidades existentes no ciclo. Um DCA completo consiste da combinação dos ciclos de vida individuais de todas as entidades. A descrição do ciclo consiste numa seqüência alternada de atividades e filas. Essa característica contribui para a modelagem de interações de entidades em que seja marcante a estrutura de filas.

Segundo Gonçalves (2004), utilizam-se dois símbolos para representar o ciclo de atividade de uma entidade, suas interações e seu estado no sistema. A figura 1 apresenta estes símbolos.

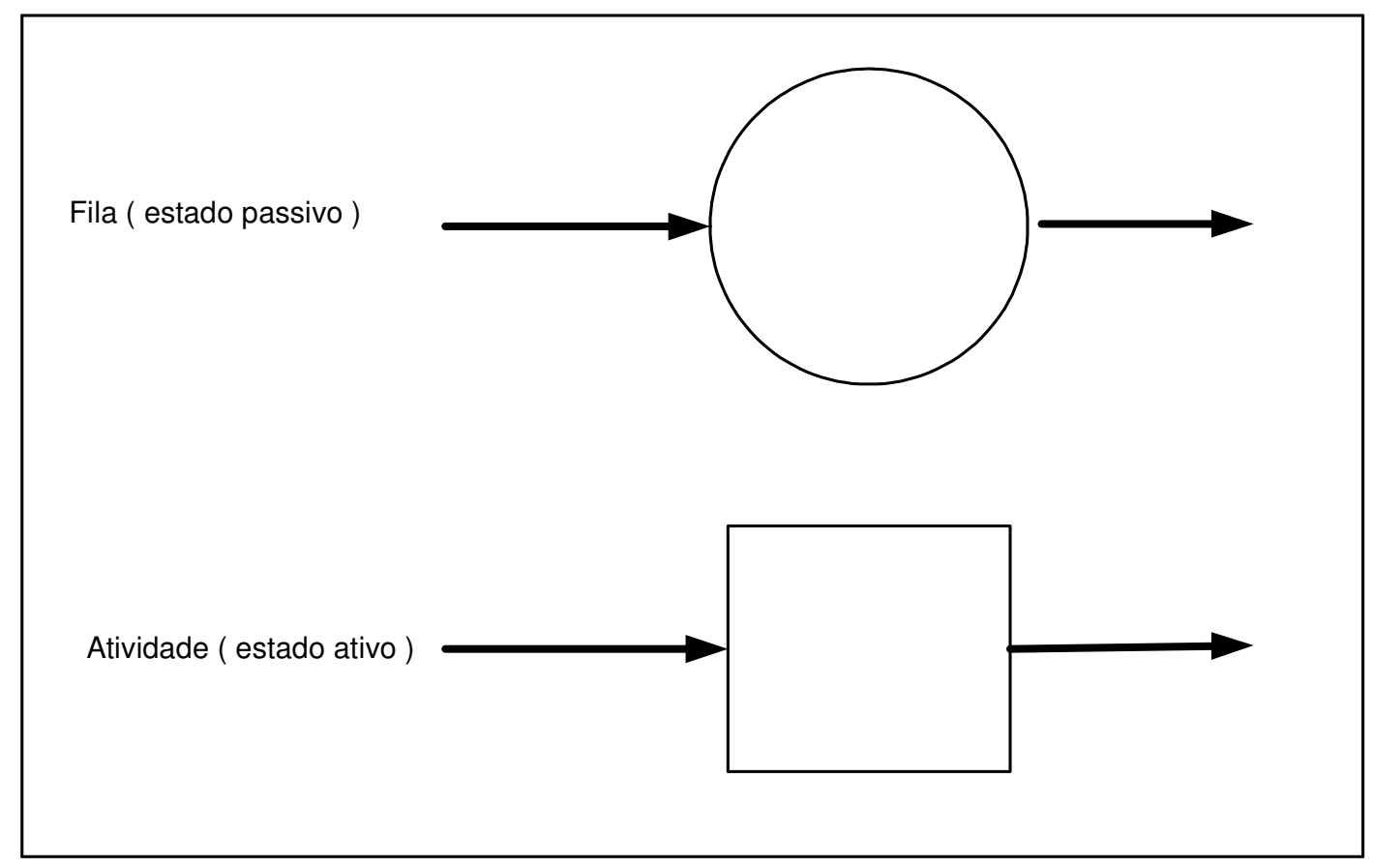

Figura 1 - Símbolos usados num DCA

Fonte: Gonçalves, 2004.

O estado passivo ocorre quando uma entidade aguarda que algum evento aconteça. Este estado representa uma fila de espera. O estado ativo representa uma atividade com um determinado tempo para sua realização. O desempenho das atividades antecessoras e sucessoras reflete no tempo de espera da entidade numa fila. 
Segundo Paul (1993) o estado ativo envolve a cooperação de diferentes classes de entidades e sua duração pode ser definida com antecedência, geralmente, utilizando-se para tal uma amostra de distribuição de probabilidade apropriada, no caso de um modelo de simulação estocástico. O estado passivo ou estado de fila envolve a não cooperação entre as diversas classes de entidades e é geralmente um estado no qual as entidades esperam algo acontecer.

Um exemplo simplificado é apresentado por Paul (1993), envolvendo três entidades num bar, que são homem, garçom e copo. $\mathrm{O}$ homem bebe ou espera para beber. $\mathrm{O}$ garçom ou serve a bebida ou fica ocioso. O copo é usado para beber ou para servir a bebida, ficando ou no estado cheio ou vazio. O DCA de cada entidade é mostrado na figura 2 a seguir:

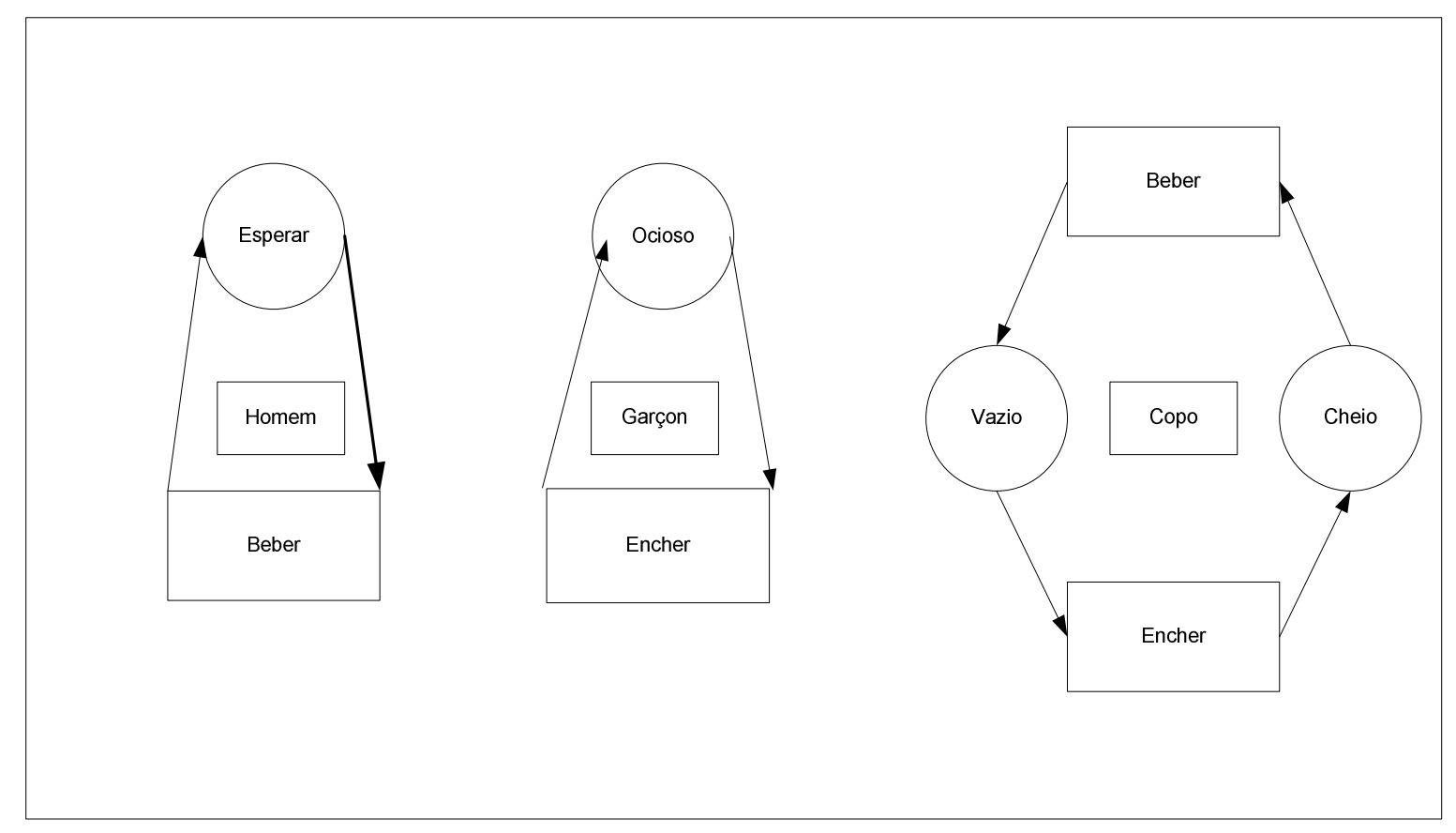

Figura 2 - DCA das entidades em um bar

Fonte: Paul, 1993. 
O DCA do bar é a representação lógica dos fluxos de atividades e das interações entre cada uma das entidades no sistema. Pelo seqüenciamento do DCA individual de cada entidade pode-se observar o diagrama de ciclo de atividade completo, conforme a figura 3:

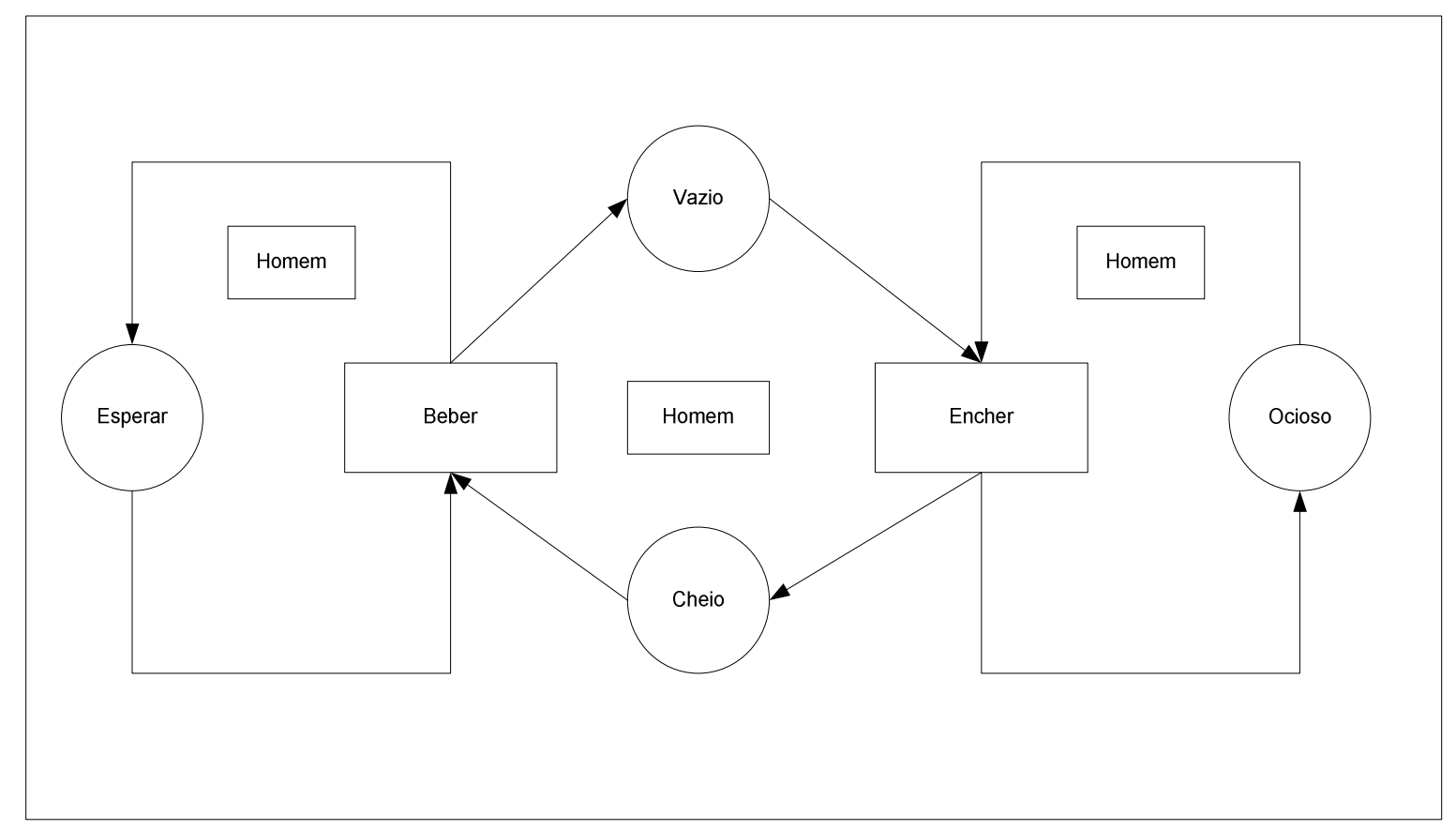

Figura 3 - DCA completo

Fonte: Paul, 1993.

Conforme se pode verificar o DCA pode ser utilizado para a compreensão do problema antes de se efetuar a modelagem da simulação, o que facilita a formulação da lógica do sistema e facilita o trabalho de desenvolvimento do modelo. Este conceito está presente na modelagem e na codificação da plataforma de simulação representando uma abordagem inovadora em termos de simulação a eventos discretos.

\section{MODELAGEM BASEADA NO CICLO DAS ENTIDADES}

Um método prático de modelagem baseada no ciclo de vida das entidades, envolvidas no modelo de simulação é proposto por De Oliveira (1999). O método é aplicado através de uma plataforma amigável que auxilia a formulação do problema e oferece uma interface para a execução do modelo por simuladores convencionais. 
A plataforma é um conjunto de módulos que fornecem as etapas básicas necessárias para a execução do experimento de simulação. As etapas mais importantes são: A formulação do problema, a geração de um código computacional e a interface com o simulador sem a necessidade de maiores esforços por parte do usuário. O método se concentra no ciclo de vida das entidades mais importantes e provê mecanismos que facilitem a edição e a criação de novas entidades. A fase de formulação é composta de três módulos:

- Entidades: captura o ciclo de vida das entidades envolvidas no sistema;

- Atividades: avalia as atividades segundo as informações fornecidas;

- Filas: verifica as filas e estabelece condições iniciais e prioridades.

\section{PLATAFORMA PARA MODELAGEM DE SIMULAÇÃO}

Uma plataforma para modelagem de simulação foi desenvolvida na UFRJ, a partir de 1980. Originalmente direcionada para o ensino de simulação, com ênfase na modelagem de problemas, foi consolidada em 1990. O sistema foi projetado no sentido de facilitar aos alunos da disciplina de simulação a difícil tarefa de formular um problema, elaborar um modelo lógico e obter resultados através de uma interface com simuladores existentes. A plataforma apresentou uma proposta inovadora, no sentido de produzir a visualização dos resultados do experimento num ambiente virtual em 3D, o que ainda não tinha sido observado em qualquer publicação desta época. No estágio atual a plataforma passa por um processo de upgrade. $\mathrm{O}$ primeiro exemplo foi desenvolvido para um hospital virtual, e será descrito a seguir.

\subsection{DESCRIÇÃO DA PLATAFORMA DE SIMULAÇÃO VISUAL}

A plataforma é constituída de módulos desenvolvidos em linguagem C++ que disponibilizam as ferramentas necessárias para o experimento de simulação. Um método prático de modelagem, baseado no ciclo de vida das entidades envolvidas no modelo, é proposto e aplicado. A plataforma provê mecanismos que facilitam a criação de novas entidades e a edição de entidades já existentes. O sistema auxilia a formulação do problema e oferece uma interface lógica para a execução da simulação. Os módulos mais importantes são descritos a seguir. 


\subsubsection{Formulação}

A formulação do problema é a etapa mais difícil e importante do experimento de simulação. Este é o passo inicial em que o usuário codifica o problema. O resultado deste módulo é código computacional com a lógica escrita em linguagem PASCAL. A formulação é composta por três elementos:

- Entidades: A funcionalidade do software oferece facilidade de edição de modo a permitir a inclusão do ciclo de uma entidade ou modificar algum atributo relacionado a uma entidade já existente, o que possibilita ao usuário navegar entre as entidades.

- Atividades: Esta funcionalidade é utilizada para checar a integridade da formulação. Se algum aspecto do problema real não foi considerado naquela etapa o usuário pode retornar ao módulo Entidades e efetuar as mudanças necessárias.

- Filas: Esta funcionalidade foi projetada para possibilitar ao usuário definir as características da fila: as condições iniciais, as distribuições estatísticas de chegada e tempos de serviço. Além disso, permite analisar todas as filas relacionadas ao ciclo de vida das entidades existentes no sistema. As figuras 4 e 5 apresentam o ciclo de vida das entidades e das atividades codificados.

\begin{tabular}{|c|c|c|c|c|c|c|c|}
\hline Arquiro & Bdicao & Binulaçao & Multisix. & Rrquivo & Sinuleçac & Mul & 28 \\
\hline & & & & CIGLO BHIOHDS & MONS is BIW & DISCIP & $S$ \\
\hline *CZCLO PAC & 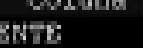 & Hotse & & PACIBNES & BSP_SAIR & BIFS & 0 \\
\hline FILA NS & & & & & BSP_C & EIFO & U \\
\hline ATIV ENTRE & & & & & ESE_RBCBE & EIFO & U \\
\hline FILA ESP_F & EEP & & & & ME & EIEO & 6 \\
\hline $\begin{array}{l}\text { ATIV RECEE } \\
\text { FILA BSP C }\end{array}$ & & & & PORTh & ESP_PACE & LIEO & \\
\hline ฉTTV cons̄ & & & & RDCEPCIONISTA & BSE_EAC & LIEO & 1 \\
\hline TILA ESP_5 & & & & MEDICO & ESE_P & LIEO & 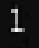 \\
\hline $\begin{array}{l}\text { ATRV SAIR } \\
\text { FILA NS }\end{array}$ & & & & SAIDA & BSP_PSA & LIFO & 1 \\
\hline
\end{tabular}

Figura 4. Ciclo de vida das entidades Codificado 


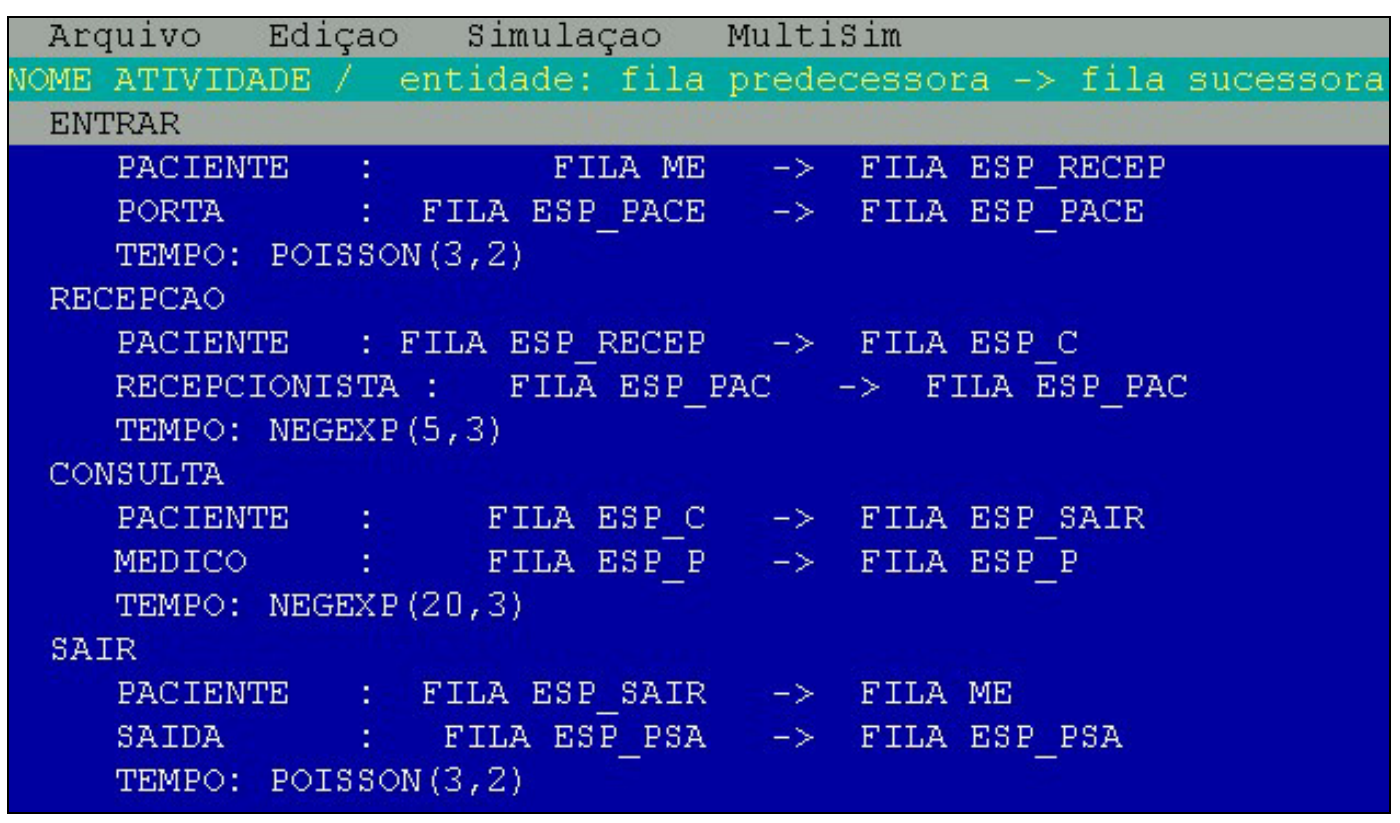

Figura 5. Ciclo de atividades Codificado

\subsubsection{Simulação}

O módulo de simulação efetua a leitura dos resultados gerados no módulo de formulação e os direciona para um software de simulação a eventos discretos de acordo com a natureza e o tipo de problema. O processo se dá a partir do programa gerador, que lê a formulação desenvolvida pelo usuário e escreve um código em PASCAL ou C++. O código PASCAL faz a interface para um pacote de simulação. O código confere flexibilidade e autosuficiência ao sistema.

\subsubsection{Simulação visual em 3d}

Este módulo utiliza as informações e o conhecimento contidos nos módulos anteriores e gera uma representação visual em $3 \mathrm{D}$, que reflete aspectos práticos do experimento. $\mathrm{O}$ usuário pode ver a simulação acontecendo em um ambiente virtual. Definem-se também as câmeras que representarão a visão de diversos usuários, as quais são colocadas em pontos estratégicos do ambiente, para possibilitar a avaliação sob diferentes perspectivas. A construção do cenário é auxiliada por computador através da utilização de CAD (computeraided design) conforme apresentado na figura 6. 


\section{Revista Produç@o}

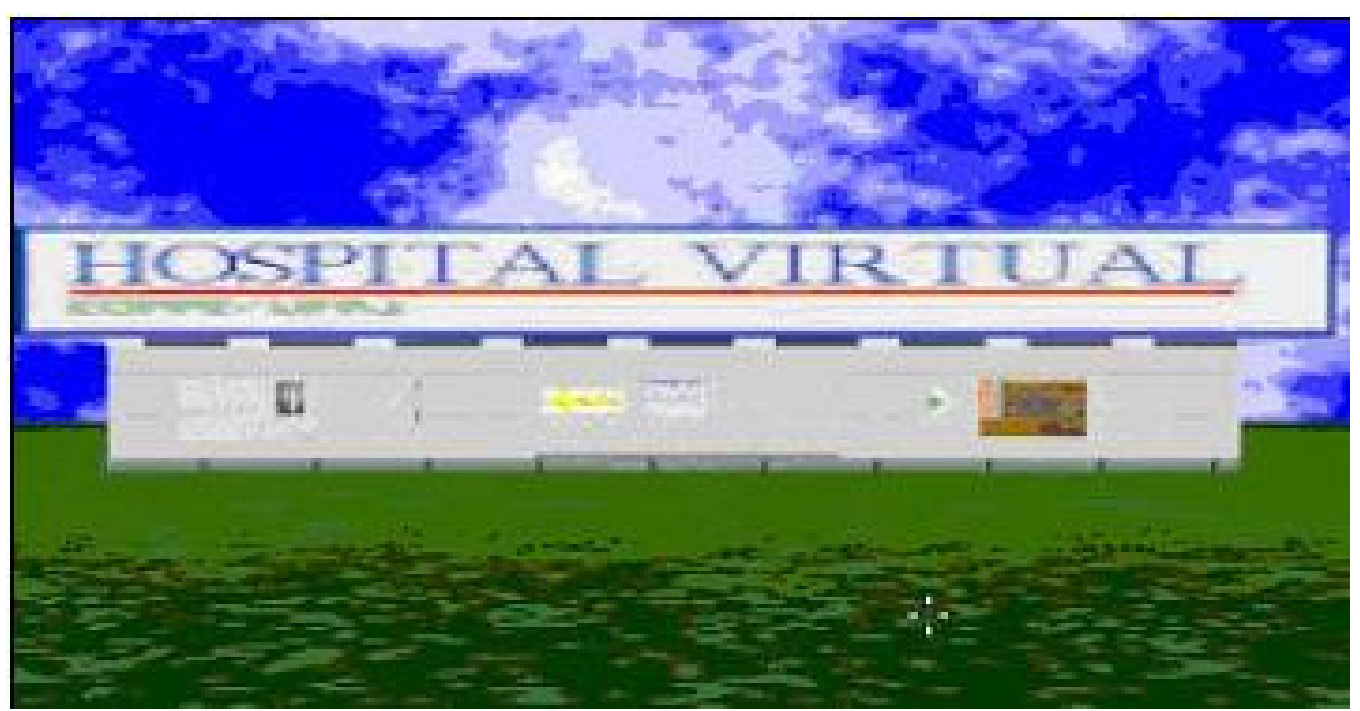

Figura 6. Um exemplo de cenário virtual utilizado pela plataforma

Fonte: De Oliveira (1999)

A simulação visual 3D é o passo final do processo de modelagem proposto. Nesta etapa são definidas as posições de todos os objetos que irão compor o cenário. Os melhores resultados da simulação são transferidos para o cenário virtual. Uma interface com a biblioteca gráfica permite que os resultados do modelo originalmente formulado possam ser animados e apresentados.

Segundo De Oliveira (1999) os principais passos numa simulação visual 3D são: a) criar os objetos; b) criar o cenário; c) aplicar textura aos objetos; d) adicionar luz; e) criar uma câmera; f) animação; e g) renderização. O processo de visualização da animação consiste basicamente na exibição de uma série de imagens, a certa velocidade, onde os objetos mudam suas posições no cenário a partir de parâmetros definidos. 


\subsubsection{Aplicações práticas}

Gaspar (1998), em sua dissertação de mestrado na COPPE/UFRJ, produziu um exemplo de uma representação 3D dos resultados teóricos da simulação de modelos de fila de espera (figura 7).
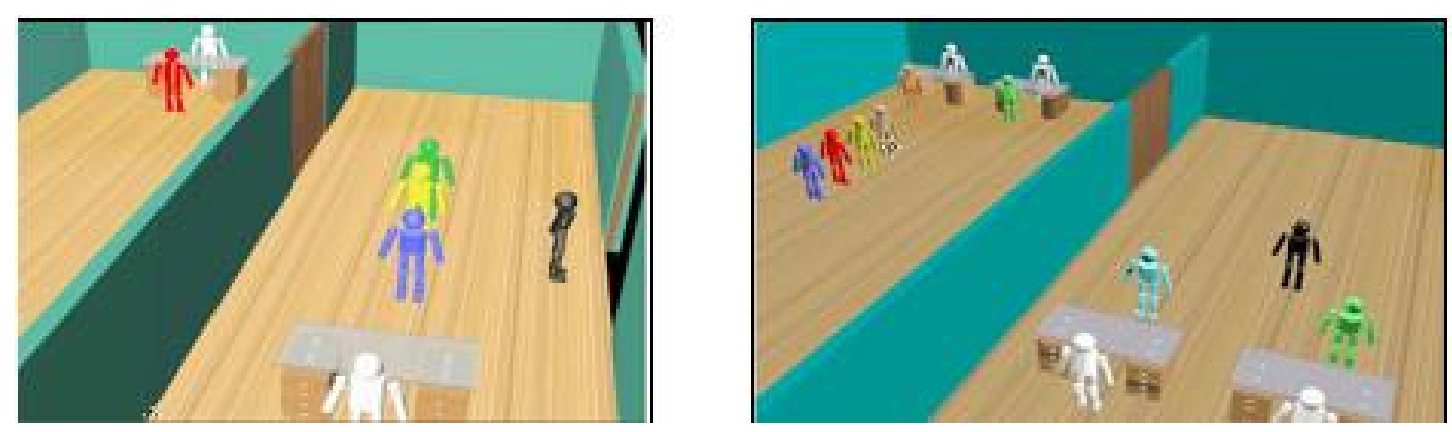

Figura 7. Simulação de filas no hospital virtual com a plataforma Fonte: Gaspar, 1998

De Oliveira e Gabcan (2002) aplicaram a simulação visual 3D para observar o fluxo de pacientes, os processos de atendimento e, também, para análise do sistema de admissão, no Instituto de Doenças do Tórax (IDT), no Hospital Universitário Clementino Fraga Filho, na Universidade Federal do Rio de Janeiro. Um exemplo prático com a utilização da plataforma proposta foi desenvolvido para a análise e avaliação de problemas de fluxo de pacientes no sistema de admissão de uma nova unidade do IDT. Os dados necessários foram coletados e um experimento de simulação completo foi realizado. 


\section{Revista Produç@o}

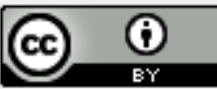

A análise da dinâmica do sistema de admissão revelou pontos críticos no layout da unidade operacional. Um cenário virtual foi desenvolvido e a simulação visual em 3D, apresentada na figura 8 , foi usada como ferramenta para auxiliar os gestores do hospital a redefinirem o sistema de admissão e melhorar o serviço disponibilizado aos pacientes. Os resultados obtidos indicaram mudanças no projeto original para de melhorar a qualidade do atendimento.

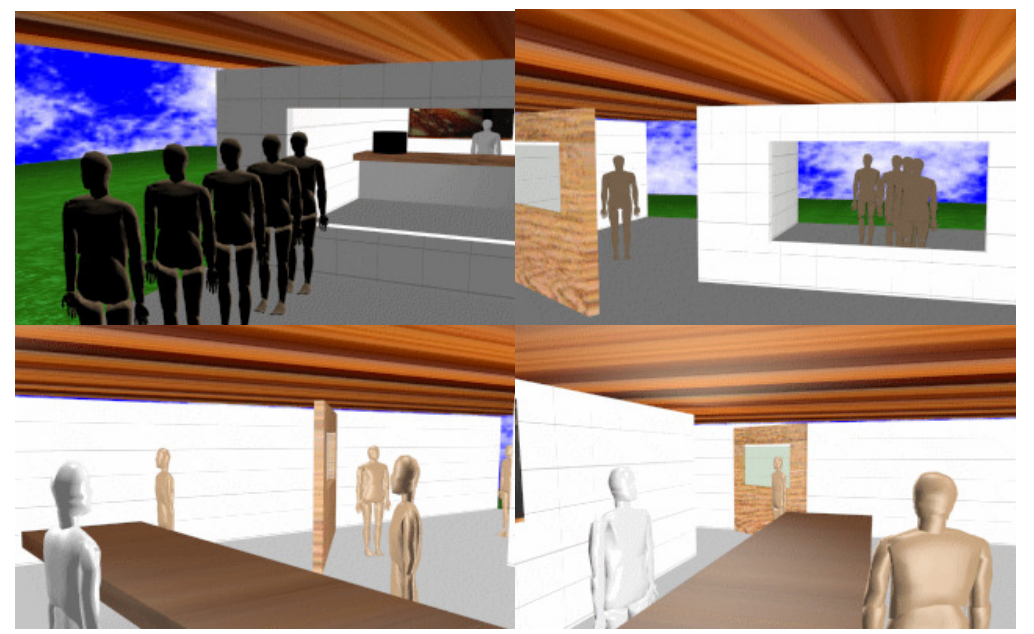

Figura 8. Simulação visual em 3D com a plataforma proposta Fonte: De Oliveira, 1999.

Moraes et al (2002) utilizaram a simulação visual 3D para estudar filas de atendimento em um hospital público considerando um ambiente multiusuário e incorporando elementos de uma nova área de aplicação que envolve conceitos de Multi-User Virtual Environment (MUVE), mostrado na figura 9.

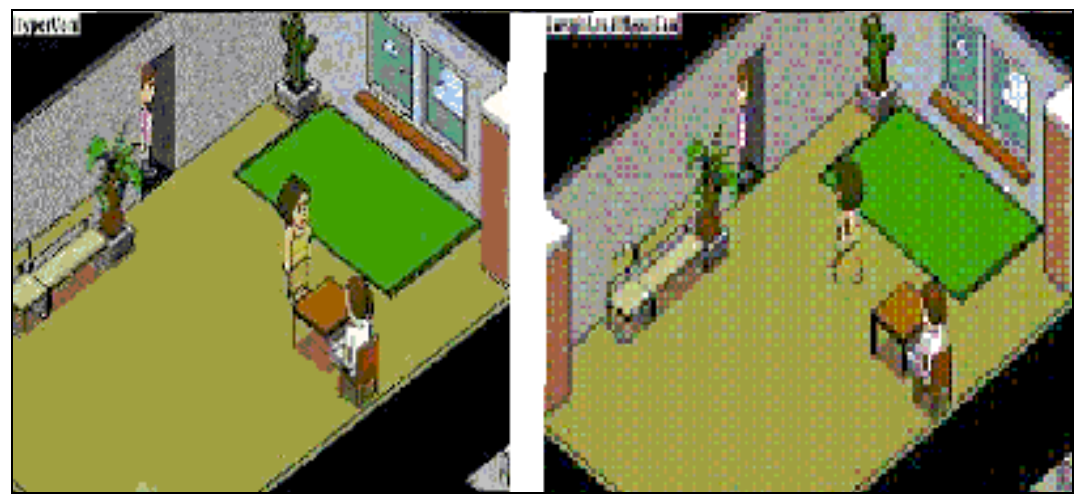

Figura 9 - Modelo de simulação multiusuário

Fonte: Moraes et al (2002) 


\section{TRABALHOS CORRELATOS}

Outras aplicações da simulação visual 3D, podem ser encontradas em áreas como manufatura, processos cirúrgicos e estudos de tráfego. $\mathrm{Na}$ manufatura podemos citar o trabalho de Mueller-Wittig et al (2002) e, também, Zhong e Yuan (2004) nos quais os autores desenvolveram uma fábrica virtual, utilizando técnicas de visualização em 3D para avaliar os resultados da simulação (figura 10).

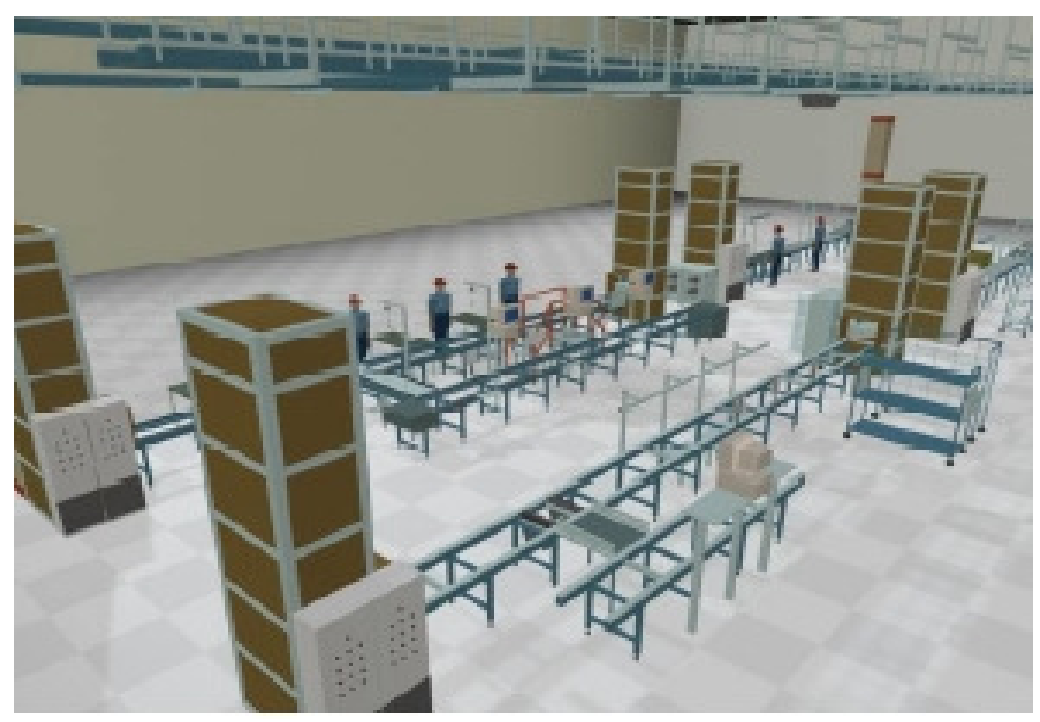

Figura 10. Linha de Montagem em 3D

Fonte: Mueller-Wittig et al. (2002)

Em estudos de tráfego, Hughes e Harkey (1999) utilizaram a simulação visual em 3D, integrando elementos de realidade virtual, para avaliar a percepção de ciclistas relacionada a fatores de risco no trânsito.

\section{CRESCIMENTO DA SIMULAÇÃO VISUAL 3D NO CENÁRIO ATUAL}

A importância crescente do tema "'Simulação visual 3D" pode ser constatada a partir da sua inclusão na pauta de temas tratados em congressos, conferências e no número de projetos em desenvolvimento. Na fase exploratória da pesquisa foram identificados alguns congressos e conferências que já destacam o tema de forma específica: 




a) WSC Winter Simulation Conference: a partir da edição de 2004 o tema simulação visual em 3D aparece agrupado com o tópico Realidade Virtual;

b) 11th International Conference on 3D Web Technology http://www.web3d2006.org/;

c) 4th International Conference on Pervasive Computing http://www.pervasive2006.org/;

d) Image 2006 - http://www.public.asu.edu/ image/.

Além disso, foram identificados, também, grupos e associações que desenvolvem pesquisas e divulgam informações sobre o tema:

a) The Image Society - The Arizona State University http://www.public.asu.edu/ image/;

b) 3D Group for Interactive Simulation - University of Rhode Island http://3dgroup.cs.uri.edu/?cat=11.

Alguns estudos nesta área vêm sendo desenvolvidos no Brasil através de projetos ou vinculados a programas de mestrado ou doutorado:

a) UFRGS - Instituto de Informática: Estudos vinculados a teses de doutorado para o desenvolvimento de ambiente de simulação visual interativa orientada a objeto;

b) UFRJ/COPPE - Desenvolvimentos através do projeto Simulação Interativa Visual, que abrange as linhas de pesquisa de simulação visual interativa multiusuário e simulação visual em 3D. Em função dos resultados alcançados estão sendo desenvolvidos estudos relacionados à simulação e realidade virtual, através de teses de mestrado e doutorado vinculadas ao projeto e que buscam a consolidação do conhecimento existente sobre a representação visual da dinâmica de modelos de simulação a eventos discretos.

\section{TENDÊNCIAS}

Algumas tendências de desenvolvimento relativas à simulação visual são: o desenvolvimento de redes de ambientes virtuais, a simulação interativa multiusuário em 
vários campos: em cirurgias; na educação, por meio de jogos; na área militar, para manuseio de máquinas e armamentos. Existe, também, a tendência de se aproximar aplicações com tecnologias de imersão em ambientes virtuais, engenharia de desempenho humano, sistemas inteligentes, aprendizado baseado em jogos, simulação baseada em agentes, linguagens de simulação (software), interoperabilidade, simulação distribuída e mundos virtuais. Neste último tópico pode-se destacar o trabalho de Moraes (2006) que propõe um experimento de simulação multiusuário para avaliar um sistema de classificação de risco, no Hospital Universitário Antônio Pedro da Universidade Federal Fluminense. O ambiente virtual multiusuário desenvolvido em 3D é mostrado na figura 11.
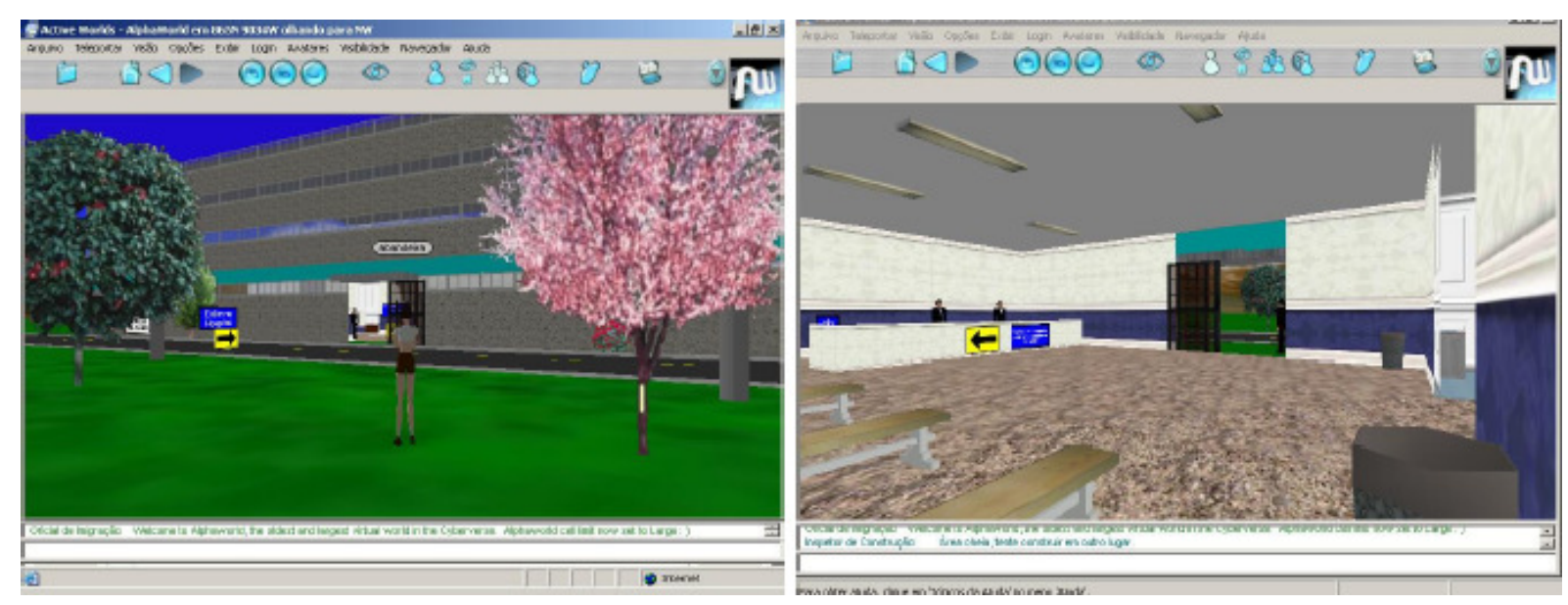

Figura 11: Hospital em 3D no Mundo Virtual

Fonte: Moraes (2006)

\section{CONCLUSÃO}

Neste artigo foi apontada a origem e a direção de desenvolvimento de uma plataforma inovadora para simulação visual em 3D, desenvolvida na COPPE/UFRJ na década de 90. Observa-se que apesar do sistema ter sido desenvolvido há mais de 20 anos atrás, sua importância pode ser constatada atualmente pelo crescente número de trabalhos que a utilizam e referenciam. O sistema foi desenvolvido numa época em que não havia muitos recursos de computação gráfica e os desdobramentos verificados acompanham o progresso tecnológico. A grande contribuição deste trabalho é ressaltar o método com ênfase no ciclo de vida das 


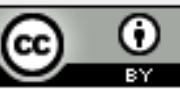

entidades, empregado pela plataforma apresentada. Esse método vem contribuindo para o desenvolvimento de muitas aplicações práticas na área social, particularmente em saúde e educação. O crescimento do interesse pelo tema foi percebido a partir da identificação de grupos, associações e do aparecimento de seções específicas em congressos e conferências nacionais e internacionais.

\section{REFERÊNCIAS}

BALCI, O. et al. The visual simulation environment technology transfer. Proceedings of the Winter Simulation Conference, 1997.

BANKS, J. \& CARSON, J. S. Discrete-event system simulation. Prentice-Hall International Series in industrial and system engineering. New Jersey: Prentice-Hall, 1984.

BELL, P.C. \& O’Keefe, R.M. Visual interactive simulation - history, recent developments and major issues, Simulation, 49, 3, (abstract). Set, 1987.

BENNETT, J.C. A flexible approach to improving outpatient clinic organization, Paper presented at 20th meeting of the Working Group OR Applied to Health Services, 31, July-4, August, Chania, Greece, 1994.

De OLIVEIRA, M.J.F. 3D Visual Simulation Platform for the Project of a New Hospital Facility. In "Monitoring, Evaluating, Planning Health Services". World Scientific Publishing Co. Pte. Ltd., ISBN 981-02-4154-2, pp 39-52, 1999.

GABCAN, L. \& De Oliveira, M.J.F. 3D visual simulation applied to new Thorax Disease Institute. In "Accessibility and Quality of Health Services". Proceedings of 28 meeting of the European Working Group on Operational Research Applied to Health Services, Rio de Janeiro, Brazil, pp 195-206, 2002.

GARCIA, L C. Dimensionamento de Recursos de Atendimento Móvel de Urgência da Região Metropolitana II do Estado do Rio de Janeiro. Dissertação de Mestrado, COPPE/UFRJ, 2006.

GASPAR, N.B. Representação Visual de Modelos de Fila de Espera. Dissertação de Mestrado, COPPE/UFRJ, 1998.

GONÇALVES, A. A. Gestão da Capacidade de Atendimento em Hospitais de Câncer. Tese de Doutorado, COPPE/UFRJ, 2004.

HUGHES, R. G. \& HARKEY, D.L. Using visual simulation to evaluate bicyclists' perceptions of selected risk factors. Chapel Hill. University of North Carolina Highway Safety Research Center, 1999. 


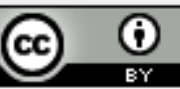

HURRION, R. D. The design, use, and required facilities of an interactive visual computer simulation language to explore production planning problem. $\mathrm{PhD}$ Thesis.Univ. of London, London, 1976.

LAGERGREN, M. Modeling as a tool to assist in managing problems in health care, In: D. Boldy, J. Braithwaite and I. Forbes (Eds.), Evidence Based Management in Health Care: The Role of Decision Support Systems, Australian Studies in Health Service Administration, 92, pp 17-36,2002

MORAES, Adriana B. et al. A multi-user simulation of a hospital queue. In Accessibility and Quality of Health Services. Proceeding of 28 meeting of the European Working Group on Operational Research Applied to Health Services, Rio de Janeiro, Brazil, pp. 195-206, 2002.

MORAES, Adriana B. Simulação multiusuário de um sistema de triagem hospitalar. Tese de Doutorado. Rio de Janeiro: COPPE-UFRJ, 2006.

MUELLER-WITTIG, W, JEGATHESE, R., SONG M., QUICK, J., WANG, H \& ZHONG, Y. VIRTUAL Factory - Highly Interactive Visualization for Manufacturing. In Proceedings of the 2002 Winter Simulation Conference, 2002. Disponível em http://www.informs-cs.org/wsc02papers/142.pdf . Acessado em 16/04/2006.

PAUL, Ray J. Activity cycle diagrams and the three Phase Method. Proceedings of the Winter Simulation Conference, 1993.

SABBADINI, F.S. Gerenciamento de restrições em hospital de emergência: um estudo de caso no hospital municipal Henrique Sergio Gregori. Dissertação de Mestrado, Universidade Estácio de Sá, 2005.

TUNICLIFE-WILSON, J.C. Review of population health care problems tackled by computer simulation, Public Health 94, pp 174-82, 1980.

WAGNER, P.E. et al. A new paradigm for visual interactive modeling and simulation. European Simulation Conference. 1996,1997. Disponível em http://www.inf.ufrgs.br/cg/publications/pwagner/opr00VEA.pdf Acesso em 08/04/2006.

ZHONG, Y. \& YUAN, X. 3D Visualization of discrete event simulation and its applications in virtual manufacturing. International Journal of CAD/CAM. Vol. 4, nr. 1, 2004. 